\title{
Review Article \\ The Case for Musical Instrument Training in Cerebral Palsy for Neurorehabilitation
}

\author{
Ana Alves-Pinto, ${ }^{1}$ Varvara Turova, ${ }^{1}$ Tobias Blumenstein, ${ }^{1}$ and Renée Lampe ${ }^{1,2}$ \\ ${ }^{1}$ Research Unit for Paediatric Neuroorthopaedics and Cerebral Palsy, Orthopedic Department, Klinikum Rechts der Isar, \\ Technical University of Munich, Ismaninger Str. 22, 81675 Munich, Germany \\ ${ }^{2}$ Markus Würth Stiftungsprofessur, Technical University of Munich, Munich, Germany
}

Correspondence should be addressed to Ana Alves-Pinto; ana.alves-pinto@tum.de

Received 24 June 2016; Accepted 29 September 2016

Academic Editor: J. Michael Wyss

Copyright (c) 2016 Ana Alves-Pinto et al. This is an open access article distributed under the Creative Commons Attribution License, which permits unrestricted use, distribution, and reproduction in any medium, provided the original work is properly cited.

\begin{abstract}
Recent imaging studies in cerebral palsy (CP) have described several brain structural changes, functional alterations, and neuroplastic processes that take place after brain injury during early development. These changes affect motor pathways as well as sensorimotor networks. Several of these changes correlate with behavioral measures of motor and sensory disability. It is now widely acknowledged that management of sensory deficits is relevant for rehabilitation in CP. Playing a musical instrument demands the coordination of hand movements with integrated auditory, visual, and tactile feedback, in a process that recruits multiple brain regions. These multiple demands during instrument playing, together with the entertaining character of music, have led to the development and investigation of music-supported therapies, especially for rehabilitation with motor disorders resulting from brain damage. We review scientific evidence that supports the use of musical instrument playing for rehabilitation in CP. We propose that active musical instrument playing may be an efficient means for triggering neuroplastic processes necessary for the development of sensorimotor skills in patients with early brain damage. We encourage experimental research on neuroplasticity and on its impact on the physical and personal development of individuals with CP.
\end{abstract}

\section{Introduction}

The central nervous system (CNS) has the ability to reorganize throughout life. This allows the CNS to adapt to changing environmental demands and to recover from injury [1]. This ability to adapt has spurred the search for new therapeutic approaches, especially in medical conditions resulting from damage to the CNS [2-4]. Also, advances in medical imaging, coupled with advances in basic neuroscience research, have stimulated the exploration of brain plasticity correlates of therapy/rehabilitation efficacy (e.g., $[5,6])$. Whilst the potential for brain plasticity is widely acknowledged, the way in which structural and functional changes in the brain are optimally triggered towards rehabilitation of motor, cognitive, and/or executive functions, as well as the mechanisms through which neuroplasticity operates, remains unclear.

One of the medical conditions that may profit from this research is cerebral palsy $(\mathrm{CP})$. In the foreground of this condition are disorders of motor function and posture resulting from injury to the CNS during development [7]. Patients may, however, also present sensory, cognitive, and attention deficits, as well as epilepsy. Despite this multisymptomatology, therapeutic programs in CP have concentrated mostly on orthopedic surgery and physical therapy to alleviate motor impairment and dysfunction [8]. Whilst this aims at giving the patients the tools to cope with demands in daily living, taking the other impairments into account in rehabilitation programs may increase the efficacy of rehabilitation training [9].

In recent years, increasing attention has been directed to the potential of music as driving vehicle for neuronal plasticity in general [10] but also in the context of neurological rehabilitation. This can be seen, for example, in the recent organization of the special research topic of "music, brain, and rehabilitation" [11]. Music has long played an important role in the therapy of children and teenagers with CP. The 
inclusion of music as therapy has been in many cases intended to increase patient's motivation or relaxation or, for example, as auditory feedback of movement [12] (passive/receptive use of music). The rhythmic properties of music have been also explored to tackle impaired gait patterns (e.g., $[13,14]$ ) through rhythmic auditory stimulation. Despite the potential of music in CP, neuroscience-based evidence of the effect of music on the rehabilitation of these patients remains scarce. Furthermore, exploration of musical instrument training for the rehabilitation of upper limb motor function is almost nonexistent and in most cases assessed only from the point of view of psychological benefits (e.g., self-confidence) and the integration benefits it brings. The current review aims at collecting and summarizing scientific evidence that supports the potential benefit that music instrument training can have not only in the improvement of motor function in patients with $C P$ but also with regard to their personal development.

We start by summarizing the main clinical characteristics of CP and alterations at the neuronal level that have been associated with specific deficits in CP. Next, we highlight the contribution of music and instrumental music to clinical rehabilitation in general with particular focus on motor disorders, selecting those that have implications for rehabilitation in CP. Finally, we review work investigating the effects of music and musical instrument training in CP. Recent reviews have covered the research on the effects of music in rehabilitation settings and on the hypothetical mechanisms underlying those effects. Our description is focused on the implications for rehabilitation in CP.

\section{Characterization of Cerebral Palsy}

A consensus definition of CP is as follows: "Cerebral Palsy describes a group of permanent disorders of the development of movement and posture, causing activity limitation, that are attributed to non-progressive disturbances that occurred in the developing fetal or infant brain. The motor disorders of CP are often accompanied by disturbances of sensation, perception, cognition, communication, and behavior, by epilepsy, and by secondary musculoskeletal problems." [15]. We now address a few of the aspects of this definition that are relevant in view of the musical instrument-based training proposed later on in this paper. More information about these and other aspects of CP can be found in Rosenbaum and Rosenbloom [9].

The first aspect refers to limitations in gross motor function. These are critical for a diagnosis of CP. Rather than falling into enclosed descriptions, these limitations can vary, depending on the onset of injury, on the areas and extension of brain structures affected, and on the factors contributing to injury $[7,15]$. Symptomatology is therefore heterogeneous among patients with CP. Nevertheless, in all cases, motor disabilities impact negatively on activities of daily living.

The second relevant aspect refers to the additional disturbances that often accompany motor limitations: disorders of sensation, perception, cognition, communication, behavior, and/or epilepsy [16-20]. These additional disturbances can occur primarily as a result of damage to brain structure and function [21-32] but can also occur in association with developmental limitations in motor function [9, 33]. Although occurrence of these additional disturbances is not decisive for the diagnosis of $\mathrm{CP}$, they are often present and they increase the complexity of the patient's clinical picture and consequently of the choice of rehabilitation activities most effective for the patient. Importantly, however, the general consensus is that these additional disabilities need also to be addressed in the clinical management of $\mathrm{CP}$, along with the motor deficits. Moreover, some deficits, for example, cognitive ones, further limit the choice of methods able to deliver effective rehabilitation.

Third, motor and additional disorders in CP result from damage to brain structures during development, pre, peri-, or postnatally, but typically before the acquisition of skills (motor, sensory, perceptual, and cognitive skills). Consequently, rehabilitation in CP needs to promote the development of nonacquired skills. This is different from the case of disorders of movement that result from brain injuries occurring in adulthood or after motor development is completed, such as stroke or Parkinson's. In these cases rehabilitation is designed to promote the recovery of previously acquired skills. The skill-learning experience of patients with $\mathrm{CP}$ is, in comparison, very different and rehabilitation needs to focus on the learning of "new" skills [9] and needs to take into account the effect of age on the development of those motor, sensory, and cognitive skills [34].

Fourth, the "nonprogressive" character of the disturbances included in the definition refers to the static character of the encephalopathy, in that neuronal damage to the CNS is permanent and does not progress with time [9]. This "nonprogressive" character however does not extend to developmental changes during childhood and the neuronal, metabolic, and body changes during adulthood that patients with CP experience and that cannot be predicted. Hence, rehabilitation programs building on the plastic potential of the brain and the continuing adaption throughout life are likely to bring effective and long-term benefit to patients.

Clinical symptoms in CP are accompanied by abnormal neuroanatomical features, detected through neuroimaging in $80-90 \%$ of the cases [35] (Table 1). Most commonly reported cases are damage to white matter pathways, especially in cases of bilateral spastic CP and athetosis [35], and to both grey and white matter structures, more frequently reported in unilateral CP [36]. Other structural abnormalities mentioned include congenital malformations, atrophy, or enlarged ventricles and cerebrospinal fluid space abnormalities, among others. Corticospinal tract projections to peripheral muscles can also be affected in CP [37]. Commonly reported in functional magnetic resonance imaging and transcranial magnetic stimulation studies in unilateral CP patients is a reorganization of central neuronal pathways, with corticomotor projections from the ipsilateral (unaffected cortex) or both motor cortices to the lesioned hand [21, 22, 29, 30, 3840] and contralateral projections to the unaffected hand [30]. $\mathrm{CP}$ patients present also a reduction in the volume of central motor structures, in comparison to controls [36, 39], reduced cortical activation during motor imagery tasks [32], and altered corticospinal pathway integrity and reduced white matter connectivity $[24,41,42]$. In addition to structural 
苟
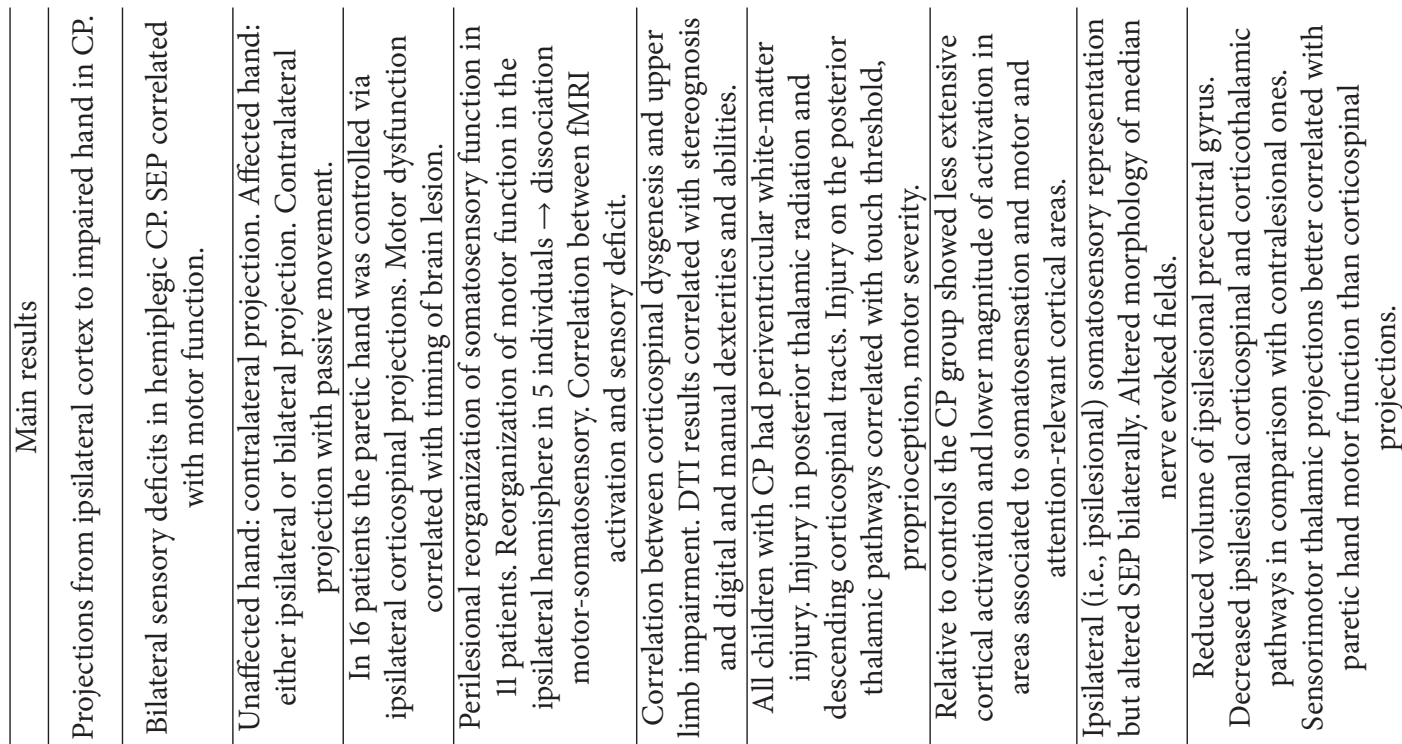

焉

0

ชें

昰

क

藏

클

ป

크

艺

क्ष

콩.

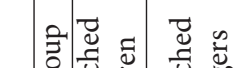

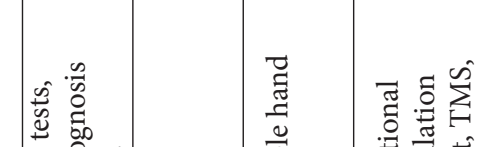

)

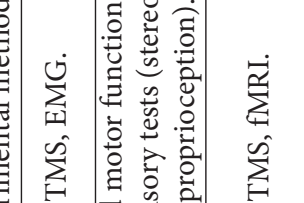

ख्रे

舒急

艺

크

กิ ฮี

莺苛

कै

㐘密.

荧

$\stackrel{5}{7}$

के

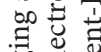

品

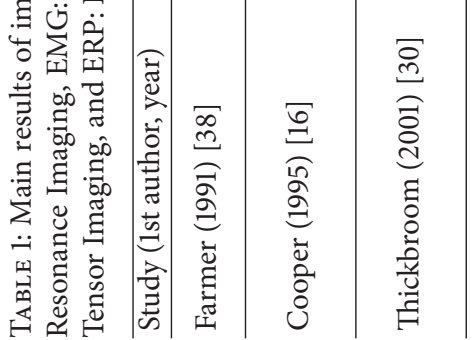

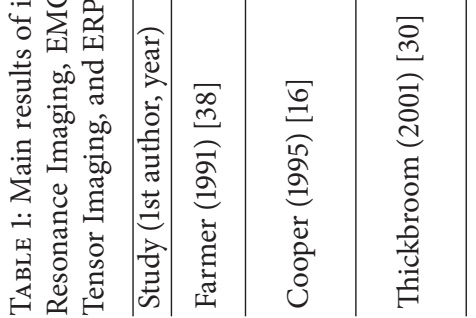

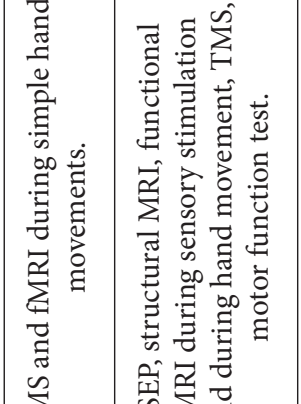

完

章范

递

t心

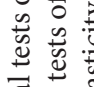

喾

ณั.

ज言

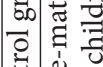

ن

齐

E

武变志

चु

a 


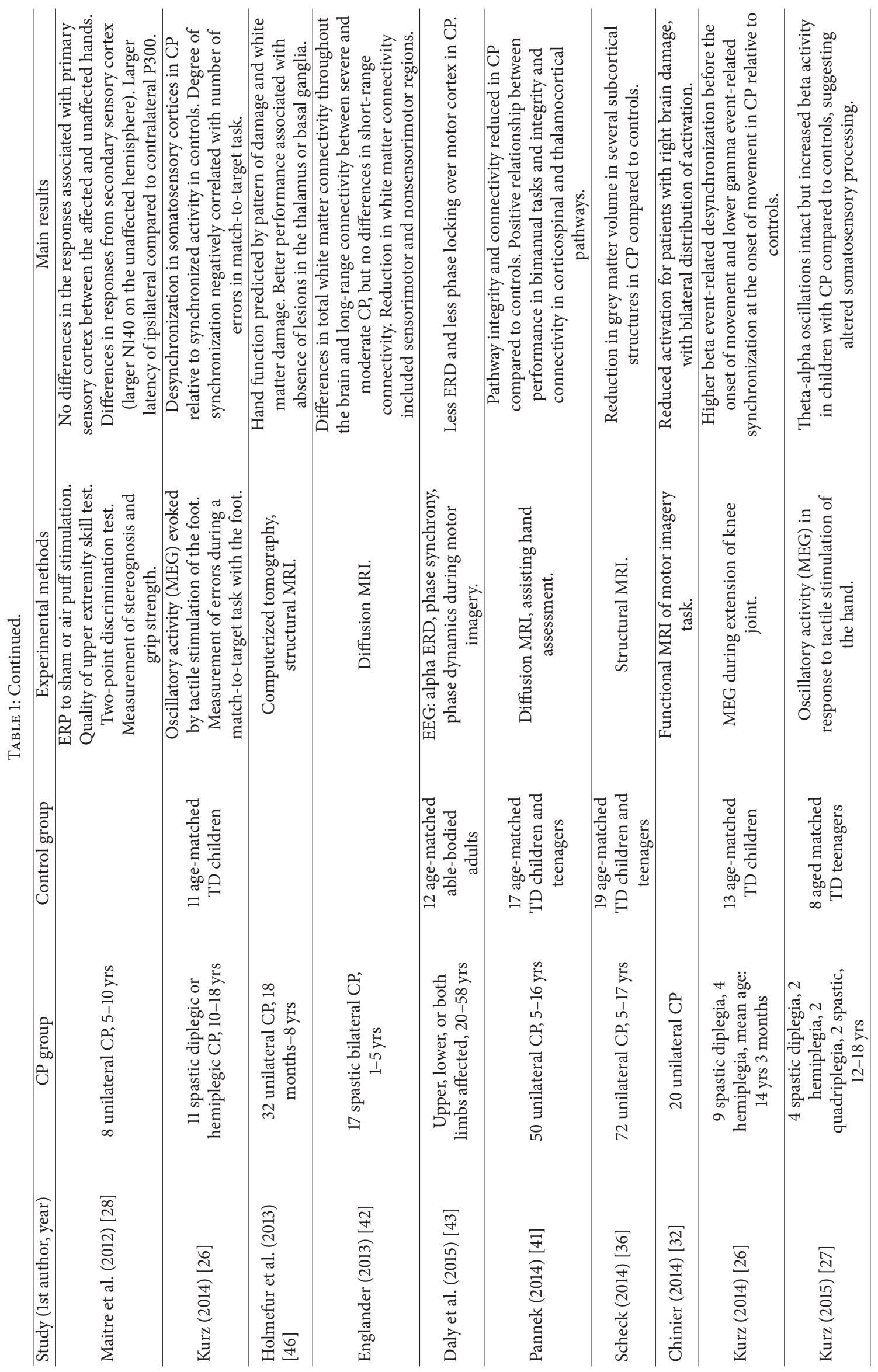


changes, movement-related neurophysiological activity is also altered in CP: magnetic resonance imaging studies have reported higher beta event-related desynchronization before movement initiation and lower gamma synchronization at the onset of movement [26], as well as lower alpha desynchronization during motor imaging [43] in patients with $\mathrm{CP}$ in comparison to controls.

However, structural alterations are not restricted to central motor tracts [21, 38, 40, 44, 47] but have also been shown to affect also sensorimotor pathways [23, 30, 31, 41, 42 , with reorganization of the latter being dissociated from the reorganization in corticospinal tracts $[22,30]$. Structural changes in sensorimotor pathways in CP are accompanied by altered somatosensory processing of tactile stimulation, with patients showing less extended cortical activation during tactile stimulation and lower magnitude of activation during tactile discrimination tasks [31]. Children with $\mathrm{CP}$ furthermore show, in comparison to typically developing peers, increased beta-activation [27] and altered activity in secondary sensory cortex in response to tactile stimulation [28]. The latter, together with the altered activation during discrimination tasks, which test the ability to distinguish between different stimuli, raises the possibility of abnormal central processing of sensory information in this clinical group and the possibility of impaired performance of motor tasks requiring the use of sensory information being due to altered sensory processing and sensorimotor interaction. Consistent with this hypothesis are the observed associations between behavioral measures of motor impairment and the degree of reorganization of sensorimotor pathways $[22,24$, $26,41]$. All in all, it is now widely accepted that clinical symptoms in CP are often not exclusively due to disturbances of the motor pyramidal pathways but can often be due to deficits in connectivity within (and outside) sensorimotor networks and in integration and processing of sensorimotor information.

\section{The Potential of Musical Instrument Training in the Development of Sensorimotor Interactions}

Against this background, training methods that demand the use of sensory information in the context of a motor task may be beneficial in conditions where sensorimotor interactions are known to be impaired. With this aim in mind, musical instrument-supported training comes up as a prime candidate for training sensorimotor interactions in $\mathrm{CP}$, since playing a musical instrument requires coordinating hand/finger movements with sensory-auditory, visual, and somatosensory-information (e.g., [10]); it involves continuous forward and backward transmission of information between different brain areas and between central and peripheral motor structures.

Effects of musical instrument training on brain structure and function have been especially investigated through imaging studies with trained musicians and nonmusicians. Studies have shown effects of long-term instrument musical training on brain plasticity both at the structural and at the functional level over several brain areas (for an in-depth review see $[10,48])$. Long-term musical practice has been associated with anatomical differences in motor and auditory cortices (larger volume, greater thickness in musicians (e.g., $[49,50]$ ), increased integrity of white matter (motor) pyramidal tracts [51], increased size of the corpus callosum [52], greater volume of cerebellum [53], and changes in multimodal integration areas (e.g., [54])). Interestingly, the type of instrument used in the training can influence the plastic mechanisms that can be measured (e.g., [54]). In addition to this, structural effects of musical training have been reported in children after 15 months of practice [55], and modulation of cortical motor outputs to the muscles involved in the performance of fine finger movements can be shaped after weeks of piano training in healthy adults [56]. Hence, musical instrument practice, and in particular piano training, has the potential to induce and promote structural and functional changes at the level of the CNS, and changes can take place at different ages (e.g., both children and adults are responsive) and at different timescales.

Beyond structural brain changes, also multimodal interactions seem to be promoted through musical instrument training and at very short time scales. In particular auditorymotor interactions have been observed in nontrained healthy adults shortly after learning a new audio-motor sequence [57] and after 20 minutes of piano training [58]. Multimodal integration seems also to be strongly promoted through musical instrument training [59], more than with auditory training only: music-associated measures of cortical plasticity were observed to be larger after musical instrument training in relation to training with auditory stimuli only [60,61]. Given the bidirectional interaction between different sensory modalities, in particular between auditory and somatosensory systems [62], it is reasonable to assume that musical training, and musical instrument training in particular, will also have an impact on this interaction and on the neuronal processing of other sensory modalities [63].

This interaction between auditory and motor systems constitutes one of the possible means through which musicsupported therapy (MST) can promote neurorehabilitation [64], especially in cases of motor disorders due to neurological damage [65] and in cases where both motor and sensorimotor networks are affected, as in CP. Even though the precise mechanisms through which damaged neuronal processes can be restored or improved remain unclear, numerous studies in the last years have shown that MST can positively influence the recovery, at least partially, of disturbed skills [59]. In one of these studies, stroke patients received regular training sessions playing MIDI piano and electronic drums (exercises adapted to individual capabilities) for three weeks [66]. After the training it was observed that both precision and smoothness of hand movements as well as the timing of movements had improved. It could also be observed that changes in oscillatory brain activity associated with motor planning (i.e., event-related desynchronization) and neuronal coherence were larger after MST than after conventional therapy [5]. MST was furthermore associated with changes in excitability in the motor cortex, derived with transcranial magnetic stimulation [6,67], and with changes in the pattern of neuronal activation, measured with 
functional imaging, in a chronic stroke patient [6]. Once again, the fact that changes could be elicited in a patient 20 months after the injury [6] suggests that plasticity is not limited to the period immediately after damage but can still be triggered, albeit with less efficacy, months after injury.

As mentioned above the way in which MST triggers changes in neuronal processes that are damaged remains unclear, in particular which aspect of music-either the rhythmic component or the pitch structure component-is essential for triggering the recovery process. In fact, some rehabilitation programs based on music therapy use the rhythmicity or periodicity of music to influence, change, or entrain movements (for a review see [64]). In a study with hemiparetic stroke patients, rhythmic auditory stimulation was employed to train new gait patterns during the first three months after injury, by the training of walking following a rhythmic pattern, using a metronome or music tapes. Increases in gait velocity and in stride length and a reduction in leg-muscle EMG-amplitude were reported in these patients, in comparison to controls [68]. Similar training procedures have also been employed to train gait patterns in Parkinson's patients (e.g., [69]) and in children and adults with $\mathrm{CP}[13,14,70]$. The fact that the training of walking using rhythmic sound properties with metronomes can influence walking kinematics in $\mathrm{CP}$ suggests that patients with $\mathrm{CP}$ can be responsive to MST-based training of movement.

\section{Music-Supported Therapy in Cerebral Palsy}

Music-based therapies are not new in the clinical management of CP. They have been typically employed to train more symmetrical and balanced gait patterns (see above) and as a vehicle for promoting motivation and emotional experience. The positive influence of music on the rehabilitation of patients with $\mathrm{CP}$ is reflected in the widespread use of music in group activities in rehabilitation and occupational day centers, where individuals typically play percussion instruments within group activities. Music is also often employed during relaxation exercises or in conjunction with other therapies with effects reported at the level of heart rate variability [71].

Less common in the rehabilitation of motor skills in CP however are MST-based methods and in particular the musical instrument training of hand motor function. In one of the few published studies, five adults with $\mathrm{CP}$ received a total of twelve sessions of musical instrument-based therapy on MIDI keyboard, twice a week for six to nine weeks [72]. The playing speed on the keyboard increased with the training to values closer to those obtained from a control group of ablebodied individuals. In another study eighteen young people (6-16 years) with CP received individual piano training with a professional piano teacher twice a week for 18 months [73]. In this case a reduction in the variability of keystroke timing was interpreted as indicating an improvement in the uniformity of keystrokes with the piano training. In an associated study, potential neuronal correlates of the effects of piano training were investigated by analyzing effective connectivity between the cerebellum and primary motor cortex in a group of ten young people with $\mathrm{CP}$ and comparing these results with those from a similar group of six that had received conventional therapy only [73]. An increase in effective connectivity from the left primary motor area to the right cerebellum in the group that received the training relative to the control group was interpreted as indicating a neuronal plastic effect resulting from piano training.

The effects of musical instrument training may however likely extend beyond motor areas and function. By recruiting the entire CNS it may likely, more than any other simple motor training, influence more strongly sensorimotor interactions leading to coordinated motor responses.

\section{Final Remarks}

To our knowledge the study of Alves-Pinto et al. [73] is the only study that has attempted to investigate the neuronal correlates of musical instrument training in $\mathrm{CP}$ and in particular in association with the training of hand motor function. Such studies are important to confirm that functional rehabilitation is not due to chance but that the therapy/training has introduced changes in the internal mechanisms underlying motor function [74]. However the validity of results can deliver false inferences if the correct experimental controls are not observed $[74,75]$. One of the difficulties for studies investigating neuroplasticity in $\mathrm{CP}$ (e.g., functional imaging) lies in the heterogeneity of this clinical group, with patients differing greatly in the symptoms presented, in the underlying brain damage, and in the onset of injury. Ways to overcome these difficulties are becoming available, especially for imaging and neurophysiological data collected in patients with CP [74].

Besides the clinical heterogeneity in $\mathrm{CP}$, additional challenges lie in the disabilities concomitant to motor impairments that are often present, namely, the learning difficulties. Besides influencing the range of experimental tests that can detect functional change induced by training, they are likely to have implications in the way musical instrument training can effectively support patients' rehabilitation. Individualized training that takes into account the specific impairments and development stage of each patient, as well as the individual training period required for plastic changes to occur, may in this case be advised in order to maximize benefit. Capturing these changes will require appropriate experimental methodology. As mentioned above, music already plays a central role in the management of patients with $\mathrm{CP}$, via music therapy-based group activities that promote personal and emotional enrichment, socializing, and relaxation. For the reasons presented above, musical instrument training, particularly active piano training on an individual basis, has the potential to trigger and promote the neuronal plastic changes and sensorimotor interactions required for rehabilitation in CP. However, experimental research is needed to validate, support, and guide the optimal use of musical instrument training in the learning of sensorimotor abilities in patients with impairments due to early brain damage.

\section{Competing Interests}

The authors report no conflict of interests in the preparation of the manuscript. 


\section{Acknowledgments}

Work was financed by the Buhl-Strohmaier Foundation.

\section{References}

[1] D. V. Buonomano and M. M. Merzenich, "Cortical plasticity: from synapses to maps," Annual Review of Neuroscience, vol. 21, no. 1, pp. 149-186, 1998.

[2] M. V. Johnston, "Clinical disorders of brain plasticity," Brain and Development, vol. 26, no. 2, pp. 73-80, 2004.

[3] M. V. Johnston, "Plasticity in the developing brain: implications for rehabilitation," Developmental Disabilities Research Reviews, vol. 15, no. 2, pp. 94-101, 2009.

[4] S. C. Cramer, M. Sur, B. H. Dobkin et al., "Harnessing neuroplasticity for clinical applications," Brain, vol. 134, no. 6, pp. 1591-1609, 2011.

[5] E. Altenmüller, J. Marco-Pallares, T. F. Münte, and S. Schneider, "Neural reorganization underlies improvement in strokeinduced motor dysfunction by music-supported therapy," Annals of the New York Academy of Sciences, vol. 1169, no. 1, pp. 395-405, 2009.

[6] N. Rojo, J. Amengual, M. Juncadella et al., "Music-supported therapy induces plasticity in the sensorimotor cortex in chronic stroke: a single-case study using multimodal imaging (fMRITMS)," Brain Injury, vol. 25, no. 7-8, pp. 787-793, 2011.

[7] E. Odding, M. E. Roebroeck, and H. J. Stam, “The epidemiology of cerebral palsy: incidence, impairments and risk factors," Disability and Rehabilitation, vol. 28, no. 4, pp. 183-191, 2006.

[8] M. L. Aisen, D. Kerkovich, J. Mast et al., "Cerebral palsy: clinical care and neurological rehabilitation," The Lancet Neurology, vol. 10, no. 9, pp. 844-852, 2011.

[9] P. L. Rosenbaum and L. Rosenbloom, Cerebral Palsy: From Diagnosis to Adult Life, John Wiley \& Sons, New York, NY, USA, 2012.

[10] S. C. Herholz and R. J. Zatorre, "Musical training as a framework for brain plasticity: behavior, function, and structure," Neuron, vol. 76, no. 3, pp. 486-502, 2012.

[11] T. Särkämö, E. Altenmüller, A. Rodríguez-Fornells, and I. Peretz, "Editorial: music, brain, and rehabilitation: emerging therapeutic applications and potential neural mechanisms," Frontiers in Human Neuroscience, vol. 10, article 103, 2016.

[12] R. P. Walmsley, L. Crichton, and D. Droog, "Music as a feedback mechanism for teaching head control to severely handicapped children: a pilot study," Developmental Medicine and Child Neurology, vol. 23, no. 6, pp. 739-746, 1981.

[13] S. J. Kim, E. E. Kwak, E. S. Park et al., "Changes in gait patterns with rhythmic auditory stimulation in adults with cerebral palsy," NeuroRehabilitation, vol. 29, no. 3, pp. 233-241, 2011.

[14] M. Thaut, C. P. Hurt, D. Dragan, and G. C. McIntosh, "Rhythmic entrainment of gait patterns in children with cerebral palsy," Developmental Medicine and Child Neurology, vol. 40, no. 78, p. 15, 1998.

[15] P. Rosenbaum et al., "Definition and classification document," Developmental Medicine and Child Neurology, vol. 49, supplement 2, pp. 8-14, 2007.

[16] J. Cooper, A. Majnemer, B. Rosenblatt, and R. Birnbaum, “The determination of sensory deficits in children with hemiplegic cerebral palsy," Journal of Child Neurology, vol. 10, no. 4, pp. 300-309, 1995.
[17] M. L. Auld, R. N. Boyd, G. L. Moseley, R. S. Ware, and L. M. Johnston, "Impact of tactile dysfunction on upper-limb motor performance in children with unilateral cerebral palsy," Archives of Physical Medicine and Rehabilitation, vol. 93, no. 4, pp. 696702, 2012.

[18] K. Clayton, J. M. Fleming, and J. Copley, "Behavioral responses to tactile stimuli in children with cerebral palsy," Physical and Occupational Therapy in Pediatrics, vol. 23, no. 1, pp. 43-62, 2003.

[19] Z. D. Jiang, X. Y. Liu, B. P. Shi, L. Lin, C. F. Bu, and A. R. Wilkinson, "Brainstem auditory outcomes and correlation with neurodevelopment after perinatal asphyxia," Pediatric Neurology, vol. 39, no. 3, pp. 189-195, 2008.

[20] T. D. Sanger and S. N. Kukke, "Abnormalities of tactile sensory function in children with dystonic and diplegic cerebral palsy," Journal of Child Neurology, vol. 22, no. 3, pp. 289-293, 2007.

[21] A. M. Gordon, Y. Bleyenheuft, and B. Steenbergen, "Pathophysiology of impaired hand function in children with unilateral cerebral palsy," Developmental Medicine and Child Neurology, vol. 55, supplement 4, pp. 32-37, 2013.

[22] A. Guzzetta, P. Bonanni, L. Biagi et al., "Reorganisation of the somatosensory system after early brain damage," Clinical Neurophysiology, vol. 118, no. 5, pp. 1110-1121, 2007.

[23] A. H. Hoon Jr., W. T. Lawrie Jr., E. R. Melhem et al., "Diffusion tensor imaging of periventricular leukomalacia shows affected sensory cortex white matter pathways," Neurology, vol. 59, no. 5, pp. 752-756, 2002.

[24] A. H. Hoon Jr., E. E. Stashinko, L. M. Nagae et al., "Sensory and motor deficits in children with cerebral palsy born preterm correlate with diffusion tensor imaging abnormalities in thalamocortical pathways," Developmental Medicine \& Child Neurology, vol. 51, no. 9, pp. 697-704, 2009.

[25] E. Pihko, P. Nevalainen, S. Vaalto et al., "Reactivity of sensorimotor oscillations is altered in children with hemiplegic cerebral palsy: a magnetoencephalographic study," Human Brain Mapping, vol. 35, no. 8, pp. 4105-4117, 2014.

[26] M. J. Kurz, E. Heinrichs-Graham, D. J. Arpin, K. M. Becker, and T. W. Wilson, "Aberrant synchrony in the somatosensory cortices predicts motor performance errors in children with cerebral palsy," Journal of Neurophysiology, vol. 111, no. 3, pp. 573-579, 2014.

[27] M. J. Kurz, E. Heinrichs-Graham, K. M. Becker, and T. W. Wilson, "The magnitude of the somatosensory cortical activity is related to the mobility and strength impairments seen in children with cerebral palsy," Journal of Neurophysiology, vol. 113, no. 9, pp. 3143-3150, 2015.

[28] N. L. Maitre, Z. P. Barnett, and A. P. F. Key, "Novel assessment of cortical response to somatosensory stimuli in children with hemiparetic cerebral palsy," Journal of Child Neurology, vol. 27, no. 10, pp. 1276-1283, 2012.

[29] M. Staudt, "Reorganization after pre- and perinatal brain lesions," Journal of Anatomy, vol. 217, no. 4, pp. 469-474, 2010.

[30] G. W. Thickbroom, M. L. Byrnes, S. A. Archer, L. Nagarajan, and F. L. Mastaglia, "Differences in sensory and motor cortical organization following brain injury early in life," Annals of Neurology, vol. 49, no. 3, pp. 320-327, 2001.

[31] J. R. Wingert, R. J. Sinclair, S. Dixit, D. L. Damiano, and H. Burton, "Somatosensory-evoked cortical activity in spastic diplegic cerebral palsy," Human Brain Mapping, vol. 31, no. 11, pp. 1772-1785, 2010.

[32] E. Chinier, S. N’Guyen, G. Lignon, A. T. Minassian, I. Richard, and M. Dinomais, "Effect of motor imagery in children with 
unilateral cerebral palsy: fMRI study," PLoS ONE, vol. 9, no. 4, Article ID e93378, 2014.

[33] A. Houwink, P. B. M. Aarts, A. C. H. Geurts, and B. Steenbergen, "A neurocognitive perspective on developmental disregard in children with hemiplegic cerebral palsy," Research in Developmental Disabilities, vol. 32, no. 6, pp. 2157-2163, 2011.

[34] G. O'Brien and L. Rosenbloom, Developmental Disability and Ageing, Mac Keith Press, London, UK, 2009.

[35] S. J. Korzeniewski, G. Birbeck, M. C. DeLano, M. J. Potchen, and N. Paneth, "A systematic review of neuroimaging for cerebral palsy," Journal of Child Neurology, vol. 23, no. 2, pp. 216-227, 2008.

[36] S. M. Scheck, K. Pannek, S. Fiori, R. N. Boyd, and S. E. Rose, "Quantitative comparison of cortical and deep grey matter in pathological subtypes of unilateral cerebral palsy," Developmental Medicine and Child Neurology, vol. 56, no. 10, pp. 968-975, 2014.

[37] A. Harrison, "Spastic cerebral palsy: possible spinal interneuronal contributions," Developmental Medicine \& Child Neurology, vol. 30, no. 6, pp. 769-780, 1988.

[38] S. F. Farmer, L. M. Harrison, D. A. Ingram, and J. A. Stephens, "Plasticity of central motor pathways in children with hemiplegic cerebral palsy," Neurology, vol. 41, no. 9, pp. 1505-1510, 1991.

[39] S. Rose, A. Guzzetta, K. Pannek, and R. Boyd, "MRI structural connectivity, disruption of primary sensorimotor pathways, and hand function in cerebral palsy," Brain Connectivity, vol. 1, no. 4, pp. 309-316, 2011.

[40] Y. Bleyenheuft, C. B. Grandin, G. Cosnard, E. Olivier, and J.L. Thonnard, "Corticospinal dysgenesis and upper-limb deficits in congenital hemiplegia: a diffusion tensor imaging study," Pediatrics, vol. 120, no. 6, pp. e1502-e1511, 2007.

[41] K. Pannek, R. N. Boyd, S. Fiori, A. Guzzetta, and S. E. Rose, "Assessment of the structural brain network reveals altered connectivity in children with unilateral cerebral palsy due to periventricular white matter lesions," NeuroImage: Clinical, vol. 5, pp. 84-92, 2014.

[42] Z. A. Englander, C. E. Pizoli, A. Batrachenko et al., "Diffuse reduction of white matter connectivity in cerebral palsy with specific vulnerability of long range fiber tracts," NeuroImage: Clinical, vol. 2, no. 1, pp. 440-447, 2013.

[43] I. Daly, J. Faller, R. Scherer et al., "Exploration of the neural correlates of cerebral palsy for sensorimotor BCI control," Frontiers in Neuroengineering, vol. 7, article 20, 2015.

[44] M. Staudt, C. Gerloff, W. Grodd, H. Holthausen, G. Niemann, and I. Krägeloh-Mann, "Reorganization in congenital hemiparesis acquired at different gestational ages," Annals of Neurology, vol. 56, no. 6, pp. 854-863, 2004.

[45] P. Nevalainen, E. Pihko, H. Mäenpää, L. Valanne, L. Nummenmaa, and L. Lauronen, "Bilateral alterations in somatosensory cortical processing in hemiplegic cerebral palsy," Developmental Medicine and Child Neurology, vol. 54, no. 4, pp. 361-367, 2012.

[46] M. Holmefur, A. Kits, J. Bergström et al., "Neuroradiology can predict the development of hand function in children with unilateral cerebral palsy," Neurorehabilitation and Neural Repair, vol. 27, no. 1, pp. 72-78, 2013.

[47] S. M. Scheck, R. N. Boyd, and S. E. Rose, "New insights into the pathology of white matter tracts in cerebral palsy from diffusion magnetic resonance imaging: a systematic review," Developmental Medicine and Child Neurology, vol. 54, no. 8, pp. 684-696, 2012.
[48] G. Schlaug, "The brain of musicians," Annals of the New York Academy of Sciences, vol. 930, no. 1, pp. 281-299, 2001.

[49] P. Bermudez, J. P. Lerch, A. C. Evans, and R. J. Zatorre, "Neuroanatomical correlates of musicianship as revealed by cortical thickness and voxel-based morphometry," Cerebral Cortex, vol. 19, no. 7, pp. 1583-1596, 2009.

[50] C. Gaser and G. Schlaug, "Brain structures differ between musicians and non-musicians," The Journal of Neuroscience, vol. 23, no. 27, pp. 9240-9245, 2003.

[51] S. L. Bengtsson, Z. Nagy, S. Skare, L. Forsman, H. Forssberg, and F. Ullén, "Extensive piano practicing has regionally specific effects on white matter development," Nature Neuroscience, vol. 8, no. 9, pp. 1148-1150, 2005.

[52] G. Schlaug, L. Jäncke, Y. Huang, J. F. Staiger, and H. Steinmetz, "Increased corpus callosum size in musicians," Neuropsychologia, vol. 33, no. 8, pp. 1047-1055, 1995.

[53] S. Hutchinson, L. H.-L. Lee, N. Gaab, and G. Schlaug, "Cerebellar volume of musicians," Cerebral Cortex, vol. 13, no. 9, pp. 943-949, 2003.

[54] M. Bangert and G. Schlaug, "Specialization of the specialized in features of external human brain morphology," European Journal of Neuroscience, vol. 24, no. 6, pp. 1832-1834, 2006.

[55] K. L. Hyde, J. Lerch, A. Norton et al., "Musical training shapes structural brain development," The Journal of Neuroscience, vol. 29, no. 10, pp. 3019-3025, 2009.

[56] A. Pascual-Leone, N. Dang, L. G. Cohen, J. P. Brasil-Neto, A. Cammarota, and M. Hallett, "Modulation of muscle responses evoked by transcranial magnetic stimulation during the acquisition of new fine motor skills," Journal of Neurophysiology, vol. 74, no. 3, pp. 1037-1045, 1995.

[57] A. Lahav, E. Saltzman, and G. Schlaug, "Action representation of sound: audiomotor recognition network while listening to newly acquired actions," The Journal of Neuroscience, vol. 27, no. 2, pp. 308-314, 2007.

[58] E. Altenmüller and M. Bangert, "Mapping perception to action in piano practice: evidence for an anterior right audio-motor interface," 2001.

[59] E. Altenmüller and G. Schlaug, "Neurobiological aspects of neurologic music therapy," Music and Medicine, vol. 5, no. 4, pp. 210-216, 2013.

[60] C. Lappe, S. C. Herholz, L. J. Trainor, and C. Pantev, "Cortical plasticity induced by short-term unimodal and multimodal musical training," Journal of Neuroscience, vol. 28, no. 39, pp. 9632-9639, 2008.

[61] C. Lappe, L. J. Trainor, S. C. Herholz, and C. Pantev, "Cortical plasticity induced by short-term multimodal musical rhythm training," PLoS ONE, vol. 6, no. 6, Article ID e21493, 2011.

[62] J. S. Butler, J. J. Foxe, I. C. Fiebelkorn, M. R. Mercier, and S. Molholm, "Multisensory representation of frequency across audition and touch: high density electrical mapping reveals early sensory-perceptual coupling," Journal of Neuroscience, vol. 32, no. 44, pp. 15338-15344, 2012.

[63] A. Kuchenbuch, E. Paraskevopoulos, S. C. Herholz, and C. Pantev, "Audio-tactile integration and the influence of musical training," PLoS ONE, vol. 9, no. 1, Article ID e85743, 2014.

[64] M. H. Thaut, G. C. McIntosh, and V. Hoemberg, "Neurobiological foundations of neurologic music therapy: rhythmic entrainment and the motor system," Frontiers in Psychology, vol. 6, article 1185, 2015.

[65] A. Rodriguez-Fornells, N. Rojo, J. L. Amengual, P. Ripollés, E. Altenmüller, and T. F. Münte, “The involvement of audio-motor 
coupling in the music-supported therapy applied to stroke patients," Annals of the New York Academy of Sciences, vol. 1252, no. 1, pp. 282-293, 2012.

[66] S. Schneider, P. W. Schönle, E. Altenmüller, and T. F. Münte, "Using musical instruments to improve motor skill recovery following a stroke," Journal of Neurology, vol. 254, no. 10, pp. 1339-1346, 2007.

[67] J. Grau-Sánchez, J. L. Amengual, N. Rojo et al., "Plasticity in the sensorimotor cortex induced by Music-supported therapy in stroke patients: a TMS study," Frontiers in Human Neuroscience, vol. 7, article 494, 2013.

[68] M. H. Thaut, G. C. McIntosh, and R. R. Rice, "Rhythmic facilitation of gait training in hemiparetic stroke rehabilitation," Journal of the Neurological Sciences, vol. 151, no. 2, pp. 207-212, 1997.

[69] M. H. Thaut, G. C. McIntosh, R. R. Rice, R. A. Miller, J. Rathbun, and J. M. Brault, "Rhythmic auditory stimulation in gait training for Parkinson's disease patients," Movement Disorders, vol. 11, no. 2, pp. 193-200, 1996.

[70] S. J. Kim, E. E. Kwak, E. S. Park, and S.-R. Cho, "Differential effects of rhythmic auditory stimulation and neurodevelopmental treatment/Bobath on gait patterns in adults with cerebral palsy: a randomized controlled trial," Clinical Rehabilitation, vol. 26, no. 10, pp. 904-914, 2012.

[71] M. Orita, N. Hayashida, T. Shinkawa et al., "Monitoring the autonomic nervous activity as the objective evaluation of music therapy for severely and multiply disabled children," The Tohoku Journal of Experimental Medicine, vol. 227, no. 3, pp. 185-189, 2012.

[72] H. J. Chong, S. Cho, E. Jeong, and S. J. Kim, "Finger exercise with keyboard playing in adults with cerebral palsy: a preliminary study," Journal of Exercise Rehabilitation, vol. 9, no. 4, pp. 420425, 2013.

[73] A. Alves-Pinto, V. Turova, T. Blumenstein, A. Thienel, A. Wohlschläger, and R. Lampe, "FMRI assessment of neuroplasticity in youths with neurodevelopmental-associated motor disorders after piano training," European Journal of Paediatric Neurology, vol. 19, no. 1, pp. 15-28, 2015.

[74] L. B. Reid, S. E. Rose, and R. N. Boyd, "Rehabilitation and neuroplasticity in children with unilateral cerebral palsy," Nature Reviews Neurology, vol. 11, no. 7, pp. 390-400, 2015.

[75] A. Eklund, T. E. Nichols, and H. Knutsson, "Cluster failure: why fMRI inferences for spatial extent have inflated false-positive rates," Proceedings of the National Academy of Sciences of the United States of America, vol. 113, no. 28, pp. 7900-7905, 2016. 

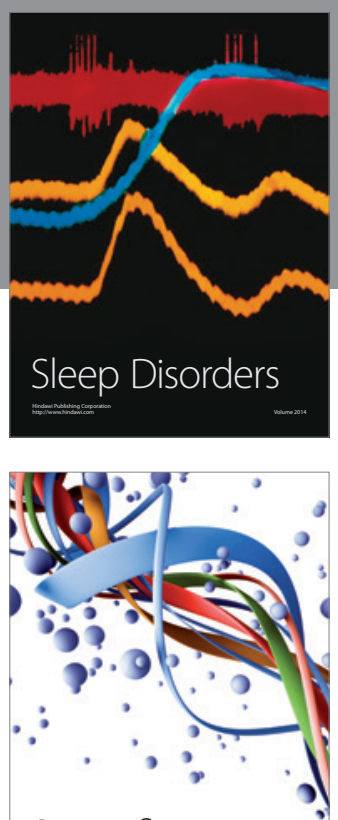

Scientifica
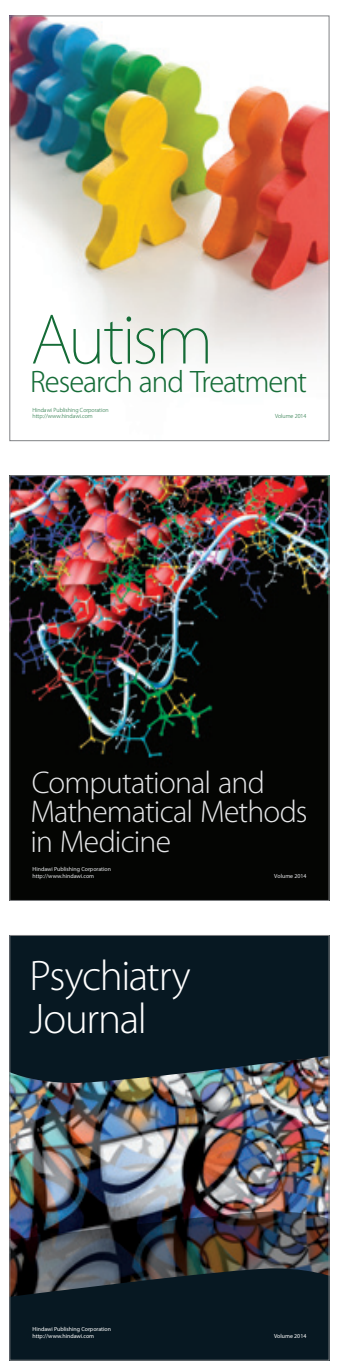
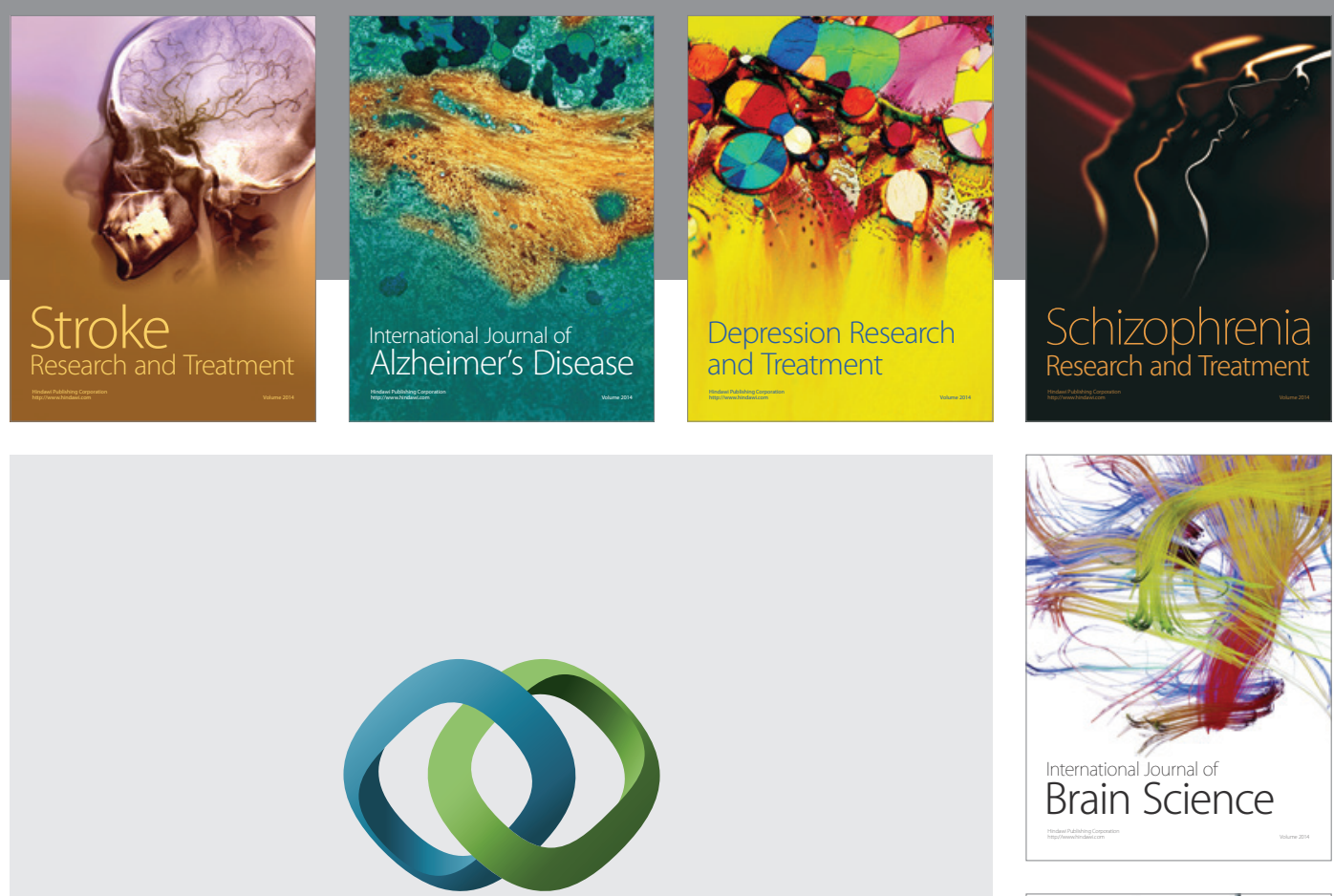

\section{Hindawi}

Submit your manuscripts at

http://www.hindawi.com
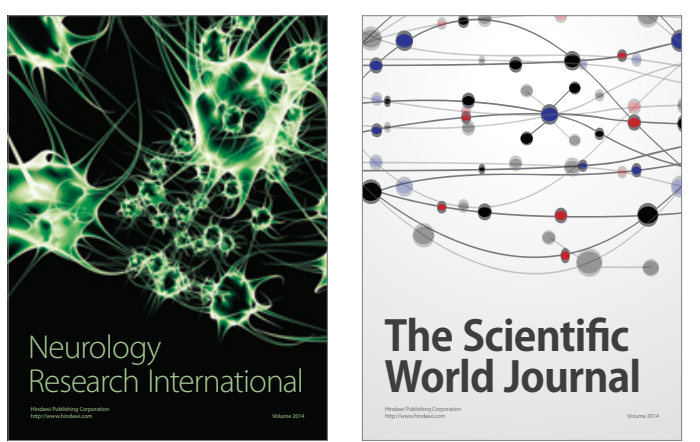

The Scientific World Journal

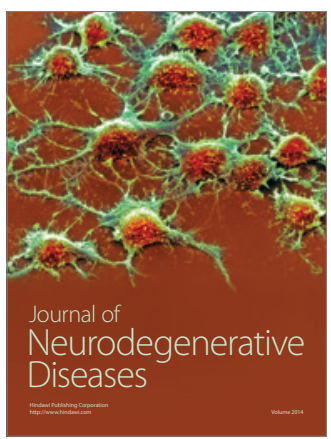

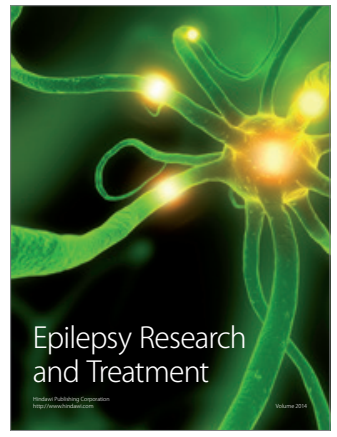

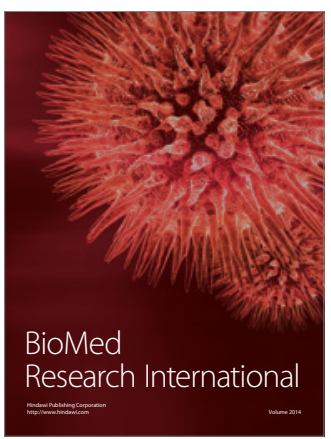

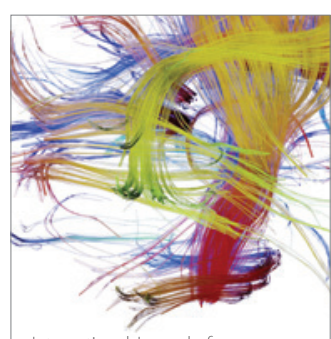

Brain Science

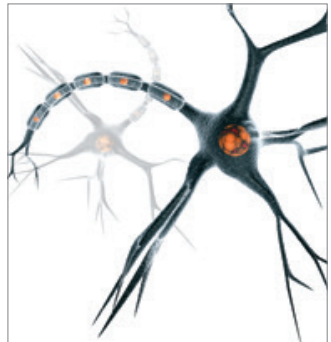

Neural Plasticity
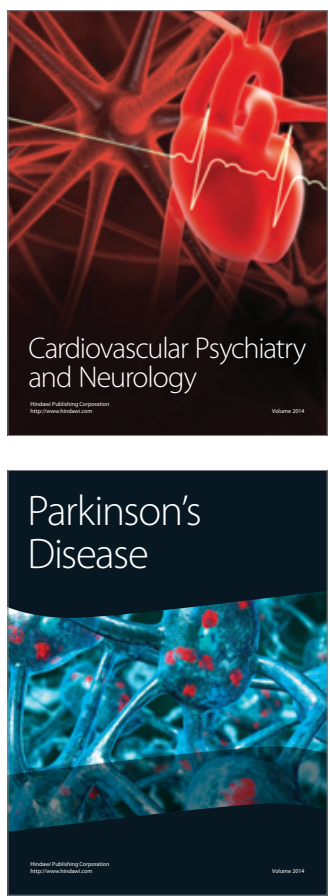